\title{
Insulin Receptor Plasma Membrane Levels Increased by the Progesterone Receptor Membrane Component 1s
}

\author{
Kaia K. Hampton, Katie Anderson, Hilaree Frazier, Olivier Thibault, and Rolf J. Craven \\ Department of Pharmacology and Nutritional Sciences, Markey Cancer Center, University of Kentucky College of Medicine, \\ Lexington, Kentucky
}

Received September 7, 2017; accepted April 13, 2018

\section{ABSTRACT}

The insulin receptor (IR) is a ligand-activated receptor tyrosine kinase that has a key role in metabolism, cellular survival, and proliferation. Progesterone receptor membrane component 1 (PGRMC1) promotes cellular signaling via receptor trafficking and is essential for some elements of tumor growth and metastasis. In the present study, we demonstrate that PGRMC1 coprecipitates with IR. Furthermore, we show that PGRMC1 increases plasma membrane IR levels in multiple cell lines and decreases insulin binding at the cell surface. The findings have therapeutic applications because a small-molecule PGRMC1 ligand, AG205, also decreases plasma membrane IR levels. However, PGRMC1 knockdown via short hairpin RNA expression and AG205 treatment potentiated insulin-mediated phosphorylation of the IR signaling mediator AKT. Finally, PGRMC1 also increased plasma membrane levels of two key glucose transporters, GLUT-4 and GLUT-1. Our data support a role for PGRMC1 maintaining plasma membrane pools of the receptor, modulating IR signaling and function.

\section{Introduction}

Changes in insulin signaling have been linked to multiple diseases, most typically diabetes (Alghamdi et al., 2014), but also loss of cognitive function (de la Monte, 2012) and cancer progression (Malaguarnera and Belfiore, 2011; Sciacca et al., 2014; Forest et al., 2015). Insulin receptor (IR) is a tetramer comprised of $2 \alpha$ (ligand binding) and $2 \beta$ (kinase domain) chains that is expressed in numerous tissues. The human IR encodes two isoforms, IR-A (lacking exon 11) and IR-B, which have a predominant role in metabolism (Belfiore et al., 2009). IR is transported in a cycle of plasma membrane export, activation, and internalization (McClain, 1992; Goh and Sorkin, 2013; Boothe et al., 2016; Posner, 2017). Signaling from the IR through the IR substrate-1/phosphatidylinositol 3-kinase/AKT pathway causes the glucose transporter (GLUT)- 4 to translocate from intracellular vesicles to the plasma membrane (Huang and Czech, 2007), increasing glucose uptake (Stöckli et al., 2011).

Progesterone receptor membrane component 1 (PGRMC1) (Cahill, 2007) contributes to signaling by stabilizing transmembrane receptors at the plasma membrane (Hampton and Craven, 2014; Thomas et al., 2014), and these receptors include tyrosine kinases (Thomas et al., 2014; Zhang et al.,

This work was supported by Washington University Diabetes Research Center Grant P30 DK020579-40; University of Kentucky CTSA Grant UL1 TR001998; and National Institutes of Health National Institute on Aging [Grant 5R01AG033649-08] and National Institutes of Health [Grant T32 DK007778 (to K.K.H. and H.F.)].

https://doi.org/10.1124/mol.117.110510.

S This article has supplemental material available at molpharm. aspetjournals.org.
2014). PGRMC1 localizes to endosomes, the endoplasmic reticulum (Nölte et al., 2000; Ahmed et al., 2010a), and the plasma membrane (Krebs et al., 2000), consistent with its highly conserved role in trafficking. In cancer, the best characterized trafficking target for PGRMC1 is the epidermal growth factor receptor (EGFR) tyrosine kinase (Ahmed et al., 2010a; Mir et al., 2012; Aizen and Thomas, 2015; Kabe et al., 2016), although PGRMC1 also increases plasma membrane pools of glucagon-like peptide- 1 receptor and membrane progestin receptor $\alpha 1$, a plasma membrane progesterone receptor (Thomas et al., 2014; Zhang et al., 2014) that may be the target for PGRMC1-dependent progesterone signaling (Bashour and Wray, 2012; Peluso, 2013; Guo et al., 2016; Sun et al., 2016).

PGRMC1 has numerous other binding partners, including cytochrome P450 proteins (Hughes et al., 2007; SzczesnaSkorupa and Kemper, 2011; Oda et al., 2011), plasminogen activator inhibitor RNA-binding protein 1 (Peluso et al., 2005, 2013), and $\alpha$-tubulin (Lodde and Peluso, 2011). PGRMC1 is attractive as a therapeutic target because it has a smallmolecule ligand, called AG-205 (Ahmed et al., 2010b; Xu et al., 2011; Zhang et al., 2014; Aizen and Thomas, 2015; Piel et al., 2016).

In the present study, we have investigated the role of PGRMC1 in maintaining IR at the plasma membrane. PGRMC1 has been associated with insulin signaling in a clinical study of insulin-resistant, high body mass index subjects, which demonstrated decreased PGRMC1 RNA levels compared with insulin-sensitive subjects (Elbein et al., 2011). We posited that downregulated PGRMC1 could disrupt normal IR function, and we show in this work that PGRMC1 has a direct role in regulating IR trafficking and signaling.

ABBREVIATIONS: Cy5, cyanine 5; EGFR, epidermal growth factor receptor; GLUT, glucose transporter; IR, insulin receptor; PBS, phosphatebuffered saline; PGRMC1, progesterone receptor membrane component 1. 


\section{Materials and Methods}

Tissue Culture. A549 cells were obtained from American Type Culture Collection (Manassas, VA) and verified by Genetica (Cincinnati, OH). HUH7 cells were provided by B. Spear (University of Kentucky College of Medicine). Cells were maintained in Dulbecco's modified Eagle's medium (Corning, Manassas, VA) containing 10\% fetal bovine serum (Sigma-Aldrich, St. Louis, MO) and antibiotics and were maintained at $37^{\circ} \mathrm{C}$ in $5 \% \mathrm{CO}_{2}$ and air. The A549 derivatives infected with lentiviruses expressing short hairpin RNAs were prepared from the plasmids pGIPZ (control) and V2LHS_90636 (PGRMC1-knockdown) and have been previously described (Ahmed et al., 2010b).

Reagents and Treatments. AG205 was purchased from Timtec (Newark, NJ). Dose response and time courses were performed previously (data not shown) to establish the most effective concentrations and times. A549 and HUH7 cells were treated with AG205 $(20 \mu \mathrm{M})$ for 90 minutes and underwent protein analysis or cell surface labeling. A549 control and PGRMC1-knockdown were plated on glassbottom microwell dishes (MatTek, Ashland, MA) for imaging. A549 control and PGRMC1-knockdown were treated with recombinant human insulin, cyanine 5 (Cy5), labeled (Nanocs, New York, NY) at a concentration of $100 \mathrm{nM}$ for 5 and 15 minutes. Cells were visualized using a Nikon $\mathrm{AlR}^{+}$resonant scanning confocal system (Nikon, Inc., Melville, NY) at the University of Kentucky Imaging Facility and analyzed with NIS-Elements C imaging software.

For cell-signaling experiments, cells were incubated in RPMI 1640 medium without serum for 6 hours. In some cases, cells were treated with vehicle control (dimethylsulfoxide) or AG-205 for the final 90 minutes of the serum starvation period. For some experiments, cells were then treated with $100 \mathrm{nM}$ recombinant human insulin (Sigma-Aldrich) for 5 minutes. In other experiments, cells were treated with $10 \mu \mathrm{M}$ erlotinib or $10 \mu \mathrm{M}$ linsitinib (Biovision, Milpitas, CA) for the final 30 minutes of serum starvation. To harvest, cells were washed with ice-cold phosphate-buffered saline (PBS) and placed on ice. The PBS was removed completely and replaced with 300-500 $\mu \mathrm{l}$ Nonidet P-40 lysis buffer (1\% Nonidet P-40, $20 \mathrm{mM}$ Tris, $150 \mathrm{mM}$ $\mathrm{NaCl}, 5 \mathrm{mM}$ EDTA, $1 \mu \mathrm{g} / \mathrm{ml}$ aprotinin, and $1 \mu \mathrm{g} / \mathrm{ml}$ leupeptin) containing phosphatase and protease inhibitors and scraped with a cell scraper. Lysates were centrifuged at 13,000 rpm to clear cell debris and assayed for protein by bicinchoninic acid assay. Immunofluorescence was performed, as previously described (Crudden et al., 2005), fixing cells in $3.7 \%$ formaldehyde and permeabilizing in $1 \%$ Triton $\mathrm{X}-100$. The anti-IR antibody was NBP2-12793 (Novus Biologicals, Littleton, CO), and cells were imaged on a Nikon TE200 microscope.

Cell Surface Labeling Assays. For a single experiment, four dishes of $90 \%-95 \%$ confluent cells were used. After the removal of media, cells were washed twice with ice-cold PBS (VWR, Radnar, PA) and labeled with sulfo-NHS-SS-biotin [sulfosuccinimidhyl-2(biotinamido)-ethyl-1,3-dithipropionate] for 30 minutes at $4^{\circ} \mathrm{C}$ on a rocking platform. The labeled proteins were purified with avidin agarose using the Cell Surface Protein Isolation Kit (Thermo Scientific, Waltham, MA), according to the manufacturer's instructions. For comparison, the intracellular protein pool that did not bind avidinagarose was also collected and stored as the unbound or cytoplasmic fraction. Cell surface labeling reactions were performed at least in triplicate, and fraction levels were confirmed via SDS-PAGE gel separation and staining with Coomassie Blue, as previously described (Ahmed et al., 2010a). Western blots of biotin-labeled eluates and unbound fractions were performed at least in triplicate.

Immunologic Techniques. For Western blot analysis, cell lysates were prepared by incubating cells in radioimmunoprecipitation assay buffer [50 mM Tris-HCl ( $\mathrm{pH} 7.4), 1 \%$ Nonidet $\mathrm{P}-40,0.25 \%$ sodium deoxycholate, $150 \mathrm{mM} \mathrm{NaCl}, 1 \mathrm{mM}$ EDTA, $1 \mathrm{mM}$ phenylmethylsulfonyl fluoride, $1 \mu \mathrm{g} / \mathrm{ml}$ aprotinin, and $1 \mu \mathrm{g} / \mathrm{ml}$ leupeptin] for 10 minutes at $4^{\circ} \mathrm{C}$. Lysates were cleared by centrifugation at $14,000 \mathrm{~g}$ for 10 minutes at $4^{\circ} \mathrm{C}$, and proteins were separated by gel electrophoresis. The antibodies used in this study were anti-IR $\beta$ (Novus Biologicals), anti-IR $\beta$ (Cell Signaling, Danvers, MA), PGRMC1 (Abcam, Cambridge, MA), anti-proliferating cell nuclear antigen (PC-10; Santa Cruz Biotechnology, Santa Cruz, CA), antiglyceraldehyde 3-phosphate dehydrogenase (Santa Cruz), anti-AKT (C67E7; Cell Signaling), anti-AKT phosphoserine 473 (Cell Signaling), anti-GLUT4 (Santa Cruz Biotechnology), and anti-GLUT1 (Santa Cruz Biotechnology). Western blots for PGRMC1 were performed with the PGR-UK1 polyclonal antibody directed to the sequence QPAASGDSDDDE of the PGRMC1 coding sequence (Ahmed et al.,
A

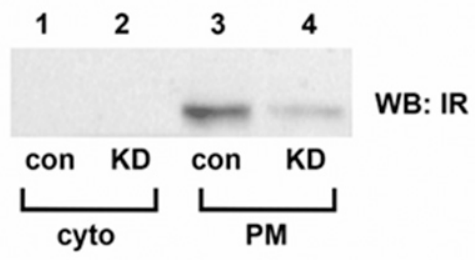

C

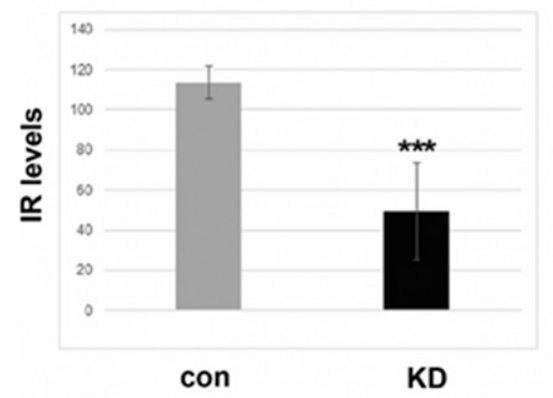

B

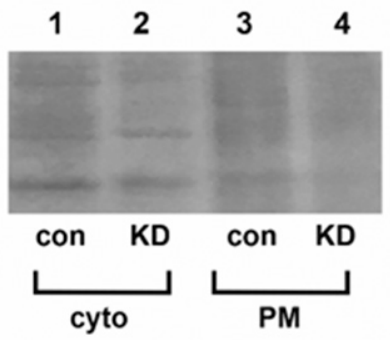

D

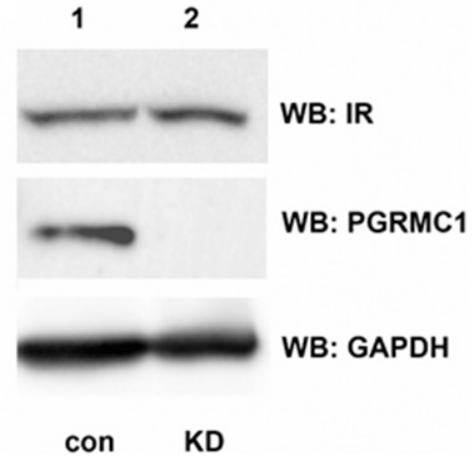

Fig. 1. PGRMC1 increases IR plasma membrane levels. (A) Western blot analysis of plasma membrane (PM) protein levels from control (con, lanes 1 and 2) and PGRMC1-knockdown cells (KD, lanes 3 and 4) labeled with biotin and purified by avidin-agarose. The Cell Signaling anti-insulin receptor $\beta$ antibody (4B8) was used to detect IR levels. Proteins that were not detected at the plasma membrane are indicative of cytoplasmic (cyto) proteins (lanes 1 and 2). The experiment was performed five separate times. (B) Coomassie-stained gel of the samples represent total protein levels. (C) Graphical representation of IR plasma membrane levels reduced in three independent replicates of knockdown (KD) versus control (con) cells $(P<$ 0.001 ). (D) Western blot analysis of total IR and PGRMC1 protein levels in control and knockdown cells. ${ }^{* * *} P<0.001$, compared with the vehicle-treated group. 
2010b). Western blots were performed at least in triplicate. For immunoprecipitations, cells were gently scraped off dishes and lysed in Nonidet P-40 buffer (described above) for 10 minutes at $4^{\circ} \mathrm{C}$. Lysates were cleared by centrifugation at $14,000 \mathrm{~g}$ for 10 minutes at $4^{\circ} \mathrm{C}$ and bound to protein A/G-agarose beads (Santa Cruz Biotechnology) containing antibody. Nonspecific antibodies matching the host species of the primary antibodies were included as negative controls. The reactions were rotated end over end at $4^{\circ} \mathrm{C}$ for 1.5 hours, centrifuged to collect precipitates, and washed three times in lysis buffer. The beads were resuspended in $1 \times$ SDS-PAGE sample loading buffer and analyzed via Western blot.

2-Deoxyglucose Uptake. For radioactive glucose uptake measurements, A549 control and PGRMC1-knockdown cells ( $5 \times 10^{5} /$ well) were plated in separate 12 -well plates in serum containing medium (Dulbecco's modified Eagle's medium) overnight. Cells were then washed twice in PBS and incubated in RPMI 1640 media (VWR) containing $1 \%$ bovine serum albumin (VWR) for 2 hours before glucose uptake studies. Cells were then washed twice with PBS and incubated in $1 \mathrm{ml}$ PBS containing $0.1 \mathrm{mM}$ 2deoxyglucose and $1 \mu \mathrm{Ci} / \mathrm{ml} 2$ deoxyD $\left[{ }^{3} \mathrm{H}\right]$ glucose (Perkin Elmer, Boston, MA) for 5 minutes at $37^{\circ} \mathrm{C}$. Cytochalasin B (20 $\mu \mathrm{M}$; Sigma-Aldrich) was added to the relevant wells with the deoxyglucose mixture to serve as a negative control. Then cells were washed three times in ice-cold PBS, solubilized in $0.4 \mathrm{ml} 1 \%$ SDS for 10 minutes at room temperature, and counted in $4 \mathrm{ml}$ Biosafe II scintillation fluid (Research Products International,
Mount Prospect, IL) for 1 minute on a Beckman LS6500 scintillation counter.

Statistics. Figures show representative blots, and each experiment was performed at least three times. Quantitation of bands was performed using Adobe Photoshop software (Adobe Systems, San Jose, CA) via histogram quantitation collected from the band, subtracted from the background. Data are expressed as mean \pm S.D. and were analyzed in Microsoft Excel using Student's paired, two-sided $t$ test to assess significance. All measurements were considered significant if $* P \leq 0.05 ; * * P \leq 0.01 ; * * * P \leq 0.001$.

\section{Results}

PGRMC1 Mediates Elevation of IR Plasma Membrane Levels. To test the role of PGRMC1 in plasma membrane stability of IR, we compared membrane levels of IR in control and PGRMC1-knockdown A549 human lung cancer cells. Extracellular proteins were biotinylated and purified with avidin column chromatography and then analyzed by Western blot using an antibody to the IR $\beta$ subunit. We will refer to IR $\beta$ as IR. IR levels decreased in PGRMC1knockdown cells relative to controls (Fig. 1A, compare lanes 3 and 4). IR was not detectable in the cytoplasmic fraction (Fig. 1A, compare lanes 1 and 2), which was diluted relative to the

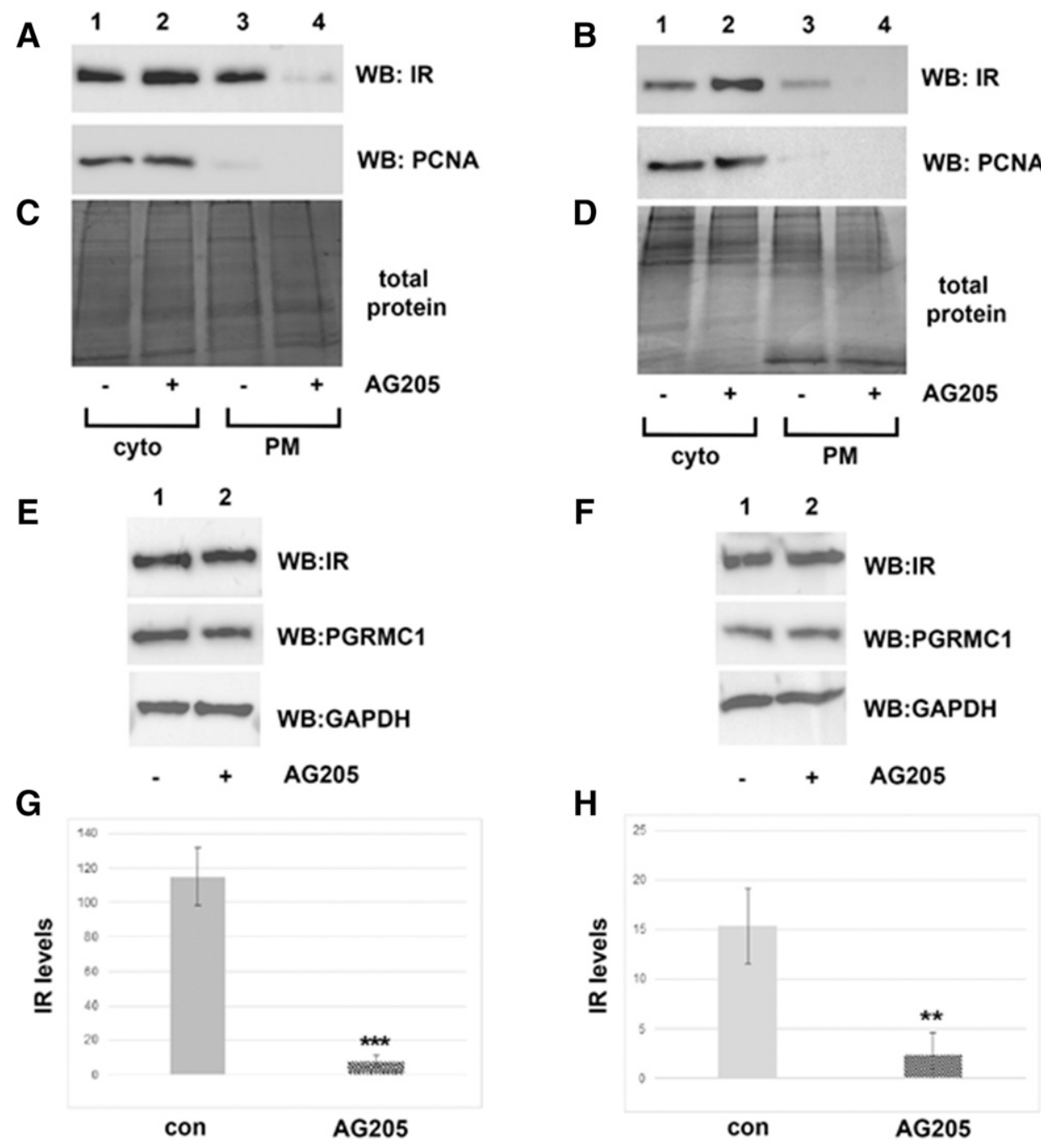

Fig. 2. Treatment with PGRMC1 ligand AG205 decreases IR plasma membrane levels. Western blot analysis of plasma membrane (PM) protein levels in A549 (A) and HUH7 (B) cell lines from control (lanes 1 and 2) and AG205 $(20 \mu \mathrm{M})$ treated (lanes 3 and 4 , respectively) labeled with biotin and purified by avidin agarose. Proteins that were not detected at the plasma membrane are indicative of cytoplasmic (cyto) proteins (lanes 1 and 2). The Novus insulin receptor $\beta$ antibody (NBP2-12793, mapping to the region between residues 1332 and 1382) was used to detect IR levels. Proliferating cell nuclear antigen serves as a control for intracellular proteins. Coomassie Blue-stained gel represents total protein levels in A549 (C) and HUH7 (D) cell lines, respectively. (E and F) Western blot analysis of total IR protein levels in A549 cells and HUH7 cells \pm AG205 treatment. IR plasma membrane levels were significantly reduced after treatment with AG205 in three independent replicates of both A549 (G) and HUH7 (H) cells $(P<0.001$; $P<0.01$, respectively). $* * P<0.01 ; * * * P<$ 0.001 compared with the vehicle-treated group. 
membrane fraction. A Coomassie brilliant Blue-stained gel of the fractions revealed few changes in band intensity (Fig. 1B). Verification of this protocol for precipitation of the cytoplasmic and plasma membrane fractions in A549 cells has been previously reported (Ahmed et al., 2010a). In multiple experiments, IR plasma membrane levels decreased 2.3-fold in PGRMC1-knockdown cells relative to controls $(P<0.005$, $t$ test; Fig. 1C). In contrast, depletion of PGRMC1 did not affect the total protein levels of IR (Fig. 1D).

In lung cancer cells, PGRMC1 is inhibited by the ligand AG205 (Ahmed et al., 2010a). To examine pharmacological inhibition of PGRMC1 on IR plasma membrane stability, we treated HUH7 human liver cancer cells, in addition to A549 human lung cancer cells, with AG205. Cell surface proteins were labeled, purified, and analyzed by Western blot, as described above. Plasma membrane levels of IR were profoundly reduced after treatment with AG205 in both cell lines (Fig. 2, A and B, compare lanes 3 and 4). The nuclear protein proliferating cell nuclear antigen served as a control for intracellular proteins (Fig. 2, A and B, compare lanes 1 and 2 ), and Coomassie Blue-stained gel of the fractions showed no variability in band intensity (Fig. 2, C and D). In multiple experiments, AG205 treatment decreased plasma membrane IR levels in both A549 and HUH7 cells by 57.5-fold and 6.8fold, respectively (Fig. 2, G and $\mathrm{H} ; P<0.005$ and $P<0.01$, respectively). As before, total cellular protein levels of IR were not affected after treatment with AG205 (Fig. 2, E and F). Together, these results suggest that PGRMC1 inhibition via AG205 treatment also decreases plasma membrane levels of IR. In these experiments, IR was detected in the unlabeled cytoplasmic fraction. This differed from the control and PGRMC1-knockdown cells shown in Fig. 1. This is likely due to the fact that two different antibodies were used for the experiments. The antibody used in Fig. 1 was generated to a common phosphorylation site, whereas the antibody used in Fig. 2 was generated to a larger epitope.

IR and PGRMC1 Coprecipitate. PGRMC1 interacts directly with the EGFR receptor tyrosine kinase and the (eight-membrane-spanning) receptor (membrane progesterone receptor alpha), suggesting that the regulation of IR by PGRMC1 could be direct. Both PGRMC1 and IR were immunoprecipitated from A549 lung cancer cells and HUH7 liver cancer cells and analyzed by Western blot. IR was efficiently precipitated (Fig. 3A, upper panel), and PGRMC1 coprecipitated with IR (Fig. 3A, lower panel) in both cell lines (lanes 2 and 4). Similarly, PGRMC1 was efficiently precipitated (Fig. 3B, upper panel), and IR coprecipitated with PGRMC1 (Fig. 3B, lower panel) in both cell lines (lanes 2 and 4). The same lysates were precipitated with a hostspecific antibody that matched to the antibodies for IR and PGRMC1 (Fig. 3, A and B, lanes 1 and 3). As an additional control, IR and PGRMC1 were precipitated from A549 control and PGRMC1-knockdown cells, and PGRMC1 was only detected in control cells (Fig. 3C, lane 2).

Cellular Binding of Insulin Dependent on PGRMC1. To determine the effect of PGRMC1 on insulin binding, A549 control and PGRMC1-knockdown cells were incubated with insulin labeled with the fluorophore Cy5 (we will refer to this as Cy5-insulin). Cy5-insulin bound readily to control A549 cells (Fig. 4C), whereas binding was largely undetectable in PGRMC1-knockdown cells (Fig. 4D). In the absence of Cy5insulin, no fluorescence was observed (Fig. 4, A and B). To better understand the pharmacodynamics of insulin binding, A549 control and PGRMC1-knockdown cells were incubated with Cy5-labeled insulin for a longer period of time (15 minutes), and real-time data were collected via video recording on
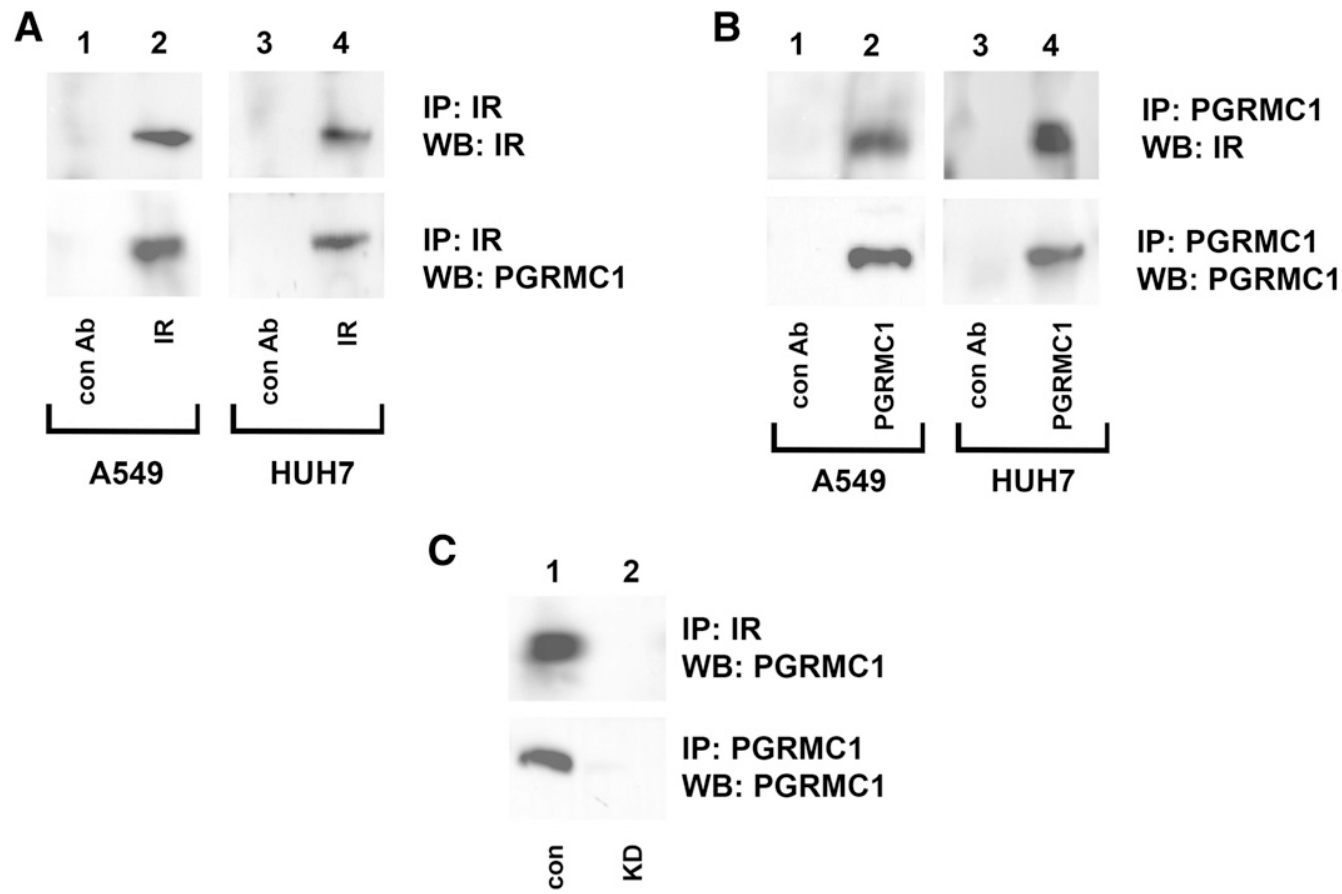

IP: IR

WB: PGRMC1

IP: PGRMC1

WB: PGRMC1

¿

Fig. 3. IR coprecipitates with PGRMC1. (A) IR and (B) PGRMC1 were immunoprecipitated from A549 cells (lanes 1 and 2) and HUH7 cells (lanes 3 and 4 ) and probed (Western blot analysis) for IR (top) or PGRMC1 (bottom). (C) IR (top panel) and PGMC1 (bottom panel) were immunoprecipitated from control (lane 1) or PGRMC1-knockdown (KD, lane 2) cells. Immunoprecipitation reactions were probed for PGRMC1 (top and bottom panels). 
A

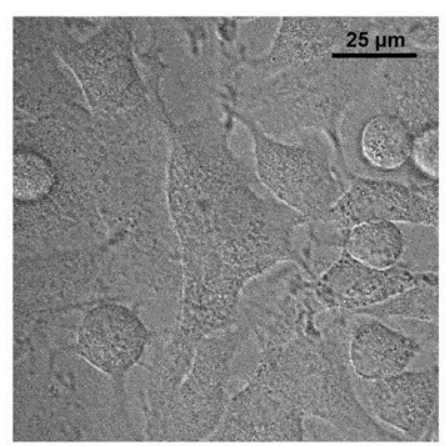

C

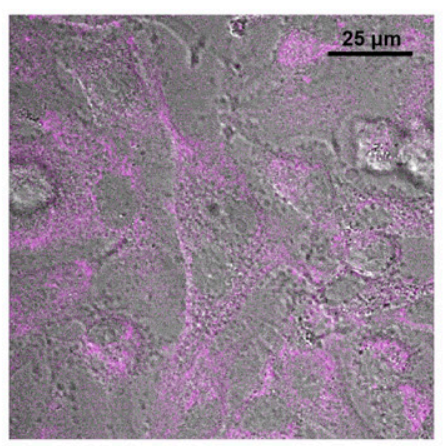

con
B

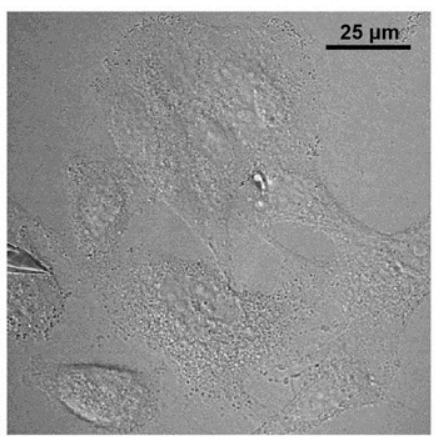

D

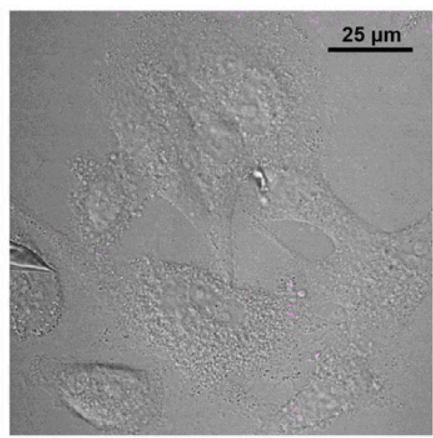

KD untr

Fig. 4. Cellular binding of insulin dependent on PGRMC1. To visualize insulin binding, control (con) and PGRMC1-knockdown (KD) A549 cells were incubated with Cy5-labeled Cy5- insulin (100 nM) and imaged. (A and B) insulin Control and PGRMC1-knockdown cells before insulin treatment (untr) and (C and D) 5 minutes after the addition of Cy5-labeled insulin. Fluorescence revealed a reduction of insulin binding in PGRMC1-knockdown cells. Images are representative of experiments performed in triplicate. (E) In a separate experiment, control and PGRMC1-knockdown cells were incubated with Cy5-labeled insulin $(100 \mathrm{nM})$ for 15 minutes. The insulin binding was recorded live (videos in Supplemental Fig. 1), and the NIS-Elements C imaging software data were exported to Excel. Insulin binding was significantly lower in PGRMC1-knockdown cells $(P<0.001, t$ test $)$.

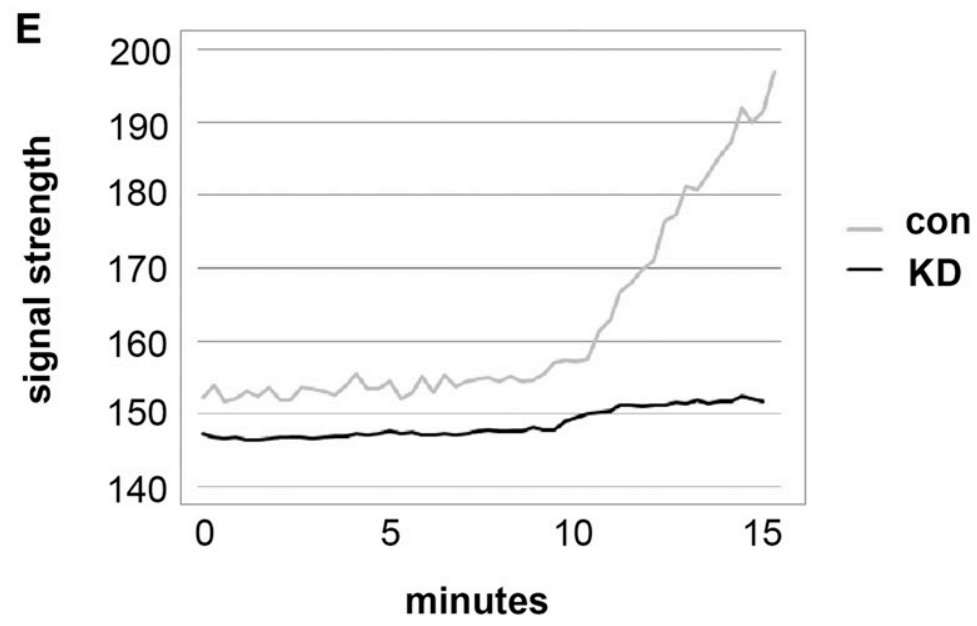

a Nikon $\mathrm{A} \mathrm{R}^{+}$confocal microscope. At the end of the time lapse, insulin binding was 10-fold higher in control cells compared with PGRMC1-knockdown cells (Fig. 4E). Thus, the results support the model that PGRMC1 mediates the interaction of IR with its ligand, insulin.

Elevated Insulin Signaling with PGRMC1 Inhibition. Because PGRMC1 increased IR plasma membrane levels and insulin binding, we determined its effect on insulin signaling. Surprisingly, AKT Ser ${ }^{473}$ phosphorylation was elevated in AG205-treated HUH7 cells (Fig. 5A, compare lanes 2 and 4), and AKT phosphorylation was increased after AG205 treatment alone (Fig. 5A, lane 3). PGRMC1 associates with IR and EGFR, but only the IR inhibitor linsitinib reversed AG205stimulated AKT phosphorylation (Fig. 5A, lane 6) and not the EGFR inhibitor erlotinib (Fig. 5A, lane 7). Insulin signaling was also potentiated by PGRMC1 inhibition in A549 cells harboring the PGRMC1 knockdown (Fig. 5B, lane 4) compared with cells expressing a control short hairpin RNA (Fig. 5B, lane 2). We posited that the combination of AG205 and insulin might result in an unusual subcellular localization of IR and performed IR immunofluorescence. Similar to the biotinylation assays in Fig. 2, the majority of IR was intracellular basally (Supplemental Fig. 1), and AG205 did not dramatically affect this localization. However, treatment with the combination of insulin and AG205 resulted in very low levels of IR staining at the cell periphery (Supplemental Fig. 1), similar to the results of the cell surface biotinylation (Fig. 2B).

PGRMC1 Mediates Glucose Transporter Plasma Membrane Levels and Facilitates Glucose Uptake. IR stimulation causes the glucose transporter GLUT-4 to 


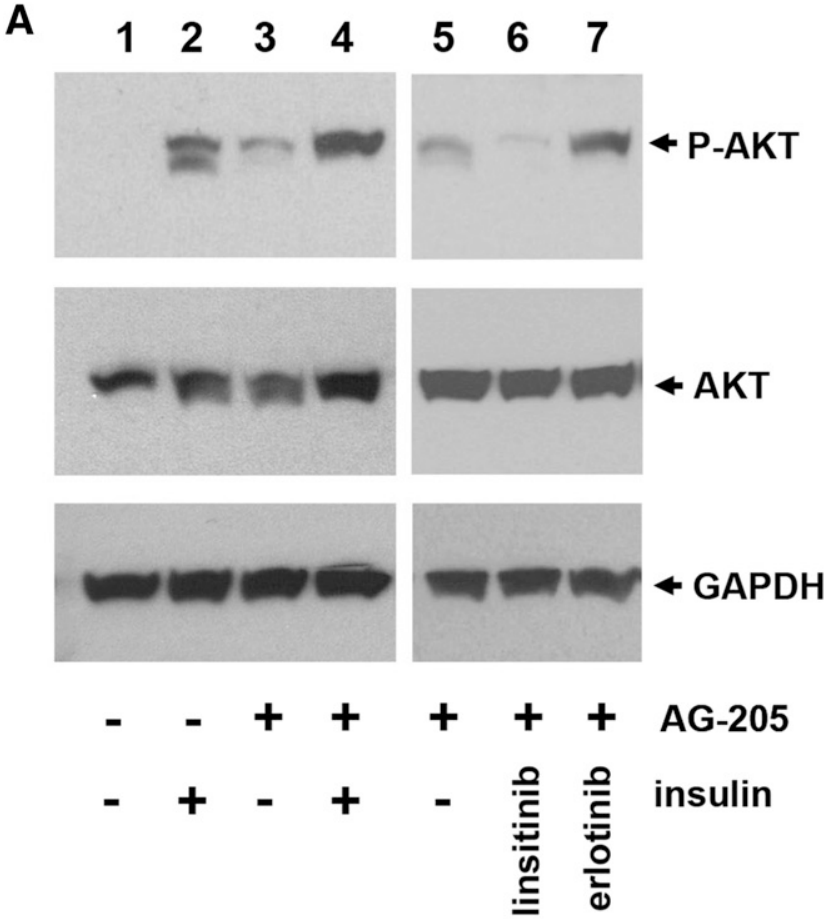

\section{B $\quad 1 \quad 2 \quad 3 \quad 4$}
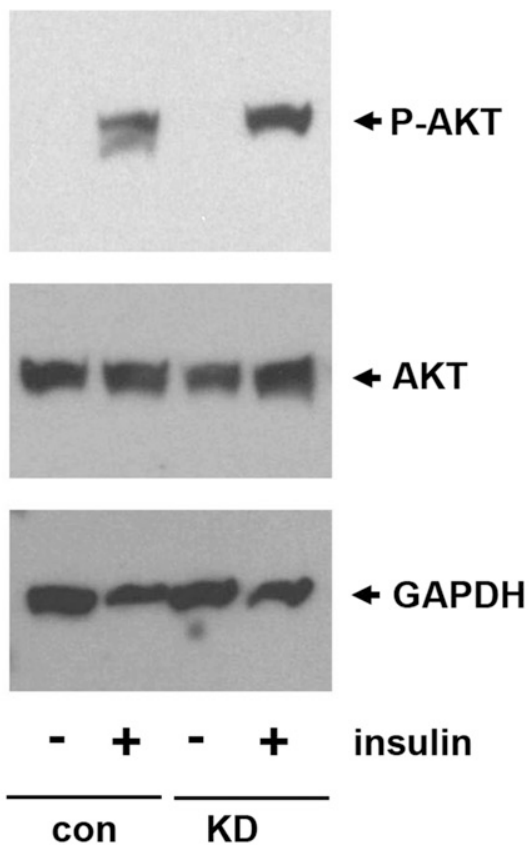

Fig. 5. Increased IR activation associated with PGRMC1 inhibition. (A) Huh-7 cells were serum starved for 6 hours. At the 4.5-hour point, vehicle control (lanes 1 and 2) or $20 \mu \mathrm{M}$ AG-205 (lanes 3-7) was added for 1.5 hours. At the end of the incubation, cells were either unstimulated (lanes 1 and 3) or treated with $100 \mathrm{nM}$ insulin (lanes 2 and 4). In lanes 5-7, cells were incubated with AG205 and a vehicle control (lane 5), $10 \mu \mathrm{M}$ linsitinib (lane 6), or $10 \mu \mathrm{M}$ erlotinib (lane 7). Cells were harvested on ice, and lysates were analyzed by Western blot for AKT-phospho-Ser ${ }^{473}$ (top panel) or total AKT. (B) A549 cells bearing a control short hairpin RNA (con, lanes 1 and 2) or a PGRMC1-knockdown short hairpin RNA (KD, lanes 3 and 4) were serum starved for 6 hours and left untreated (lanes 1 and 3 ) or were treated with $100 \mathrm{nM}$ insulin (lanes 2 and 4) for 5 minutes and harvested. Lysates were analyzed, as described in (A). translocate from intracellular vesicles to the plasma membrane (Pessin et al., 1999; Huang and Czech, 2007). Because PGRMC1 elevated IR plasma membrane levels, we posited that it would also increase plasma membrane GLUT-4. Indeed, plasma membrane GLUT-4 levels declined in PGRMC1-knockdown cells (Fig. 6A, upper panel). Interestingly, the levels of the constitutive glucose transporter, GLUT1, also declined in PGRMC1-knockdown cells, although to a lesser extent (Fig. 6A, lower panel). In triplicate experiments, the decreases in GLUT-4 and GLUT-1 in A549/ribonucleic acid interference cells were 2.6 -fold $(P<0.01)$ and 1.5 -fold $(P$ $=0.02$ ), respectively (Fig. $6 \mathrm{~B})$. The total protein levels of GLUT-4 and GLUT-1 did not change between control and PGRMC1-knockdown cells (Fig. 6C).

Because PGRMC1 increased the levels of glucose transporters at the plasma membrane, we tested whether glucose transport was also affected. A549 control and PGRMC1knockdown cells were incubated with ${ }^{3} \mathrm{H}$-glucose and washed extensively. PGRMC1-knockdown cells exhibited a 1.5-fold decrease in basal uptake of ${ }^{3} \mathrm{H}$-glucose compared with control cells (Fig. 6D, $P=0.0007, t$ test). The actin polymerization inhibitor cytochalasin $B$, which arrests the transport of glucose transporters to the plasma membrane, served as a negative control.

\section{Discussion}

IR plays a crucial role in metabolism and performs key functions in the muscle, fat, liver, and brain (Saltiel and Kahn, 2001). IR is also overexpressed in cancer (Papa et al., 1990), where it has a ligand-dependent transforming activity in fibroblasts (Giorgino et al., 1991). Cancer cells typically express the IR-A form (Frasca et al., 1999), which is also expressed in embryonic tissues and in the brain, and differs from the IR-B isoform, which has an additional 12 amino acids due to a splice variant in exon 11 (Moller et al., 1989; Mosthaf et al., 1990). IR-A acts as a receptor for insulin-like growth factor-II, which is induced in cancer, with equal affinity to insulin-like growth factor-1 receptor (Frasca et al., 1999), suggesting that IR directs tumor-specific signaling that promotes metabolism.

The current work demonstrates a key role for PGRMC1 in maintaining IR at the plasma membrane. We primarily used A549 cells because we have a verified and well-characterized ribonucleic acid interference-mediated knockdown model system in A549 cells (Ahmed et al., 2010a,b; Mir et al., 2012). A549 cells express IR, and they are a model system for IR signaling (Jones et al., 2014). IR increases proliferation and therapeutic resistance in this non-small cell lung cancer cell line (Vincent et al., 2013; Forest et al., 2015; Franks et al., 2016). Our group previously showed that PGRMC1 is essential for trafficking the EGFR receptor tyrosine kinase in cancer cells and is associated with EGFR (Ahmed et al., 2010a). This finding was elegantly extended by Kabe et al. (2016), who showed that PGRMC1 forms heme-dependent dimers that associate with EGFR, driving downstream signaling, cell transformation, and tumor metastasis. We report in this work that PGRMC1 also associates with IR and increases IR plasma membrane levels. Future research will determine whether PGRMC1 complexes with IR are also heme-dependent. 
A

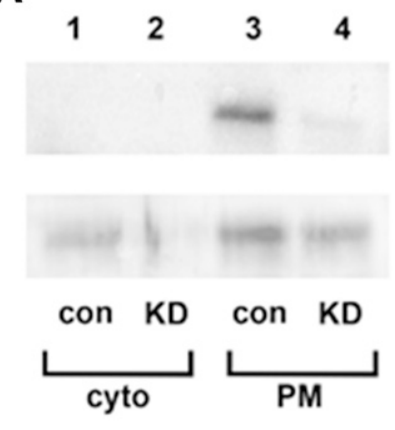

B
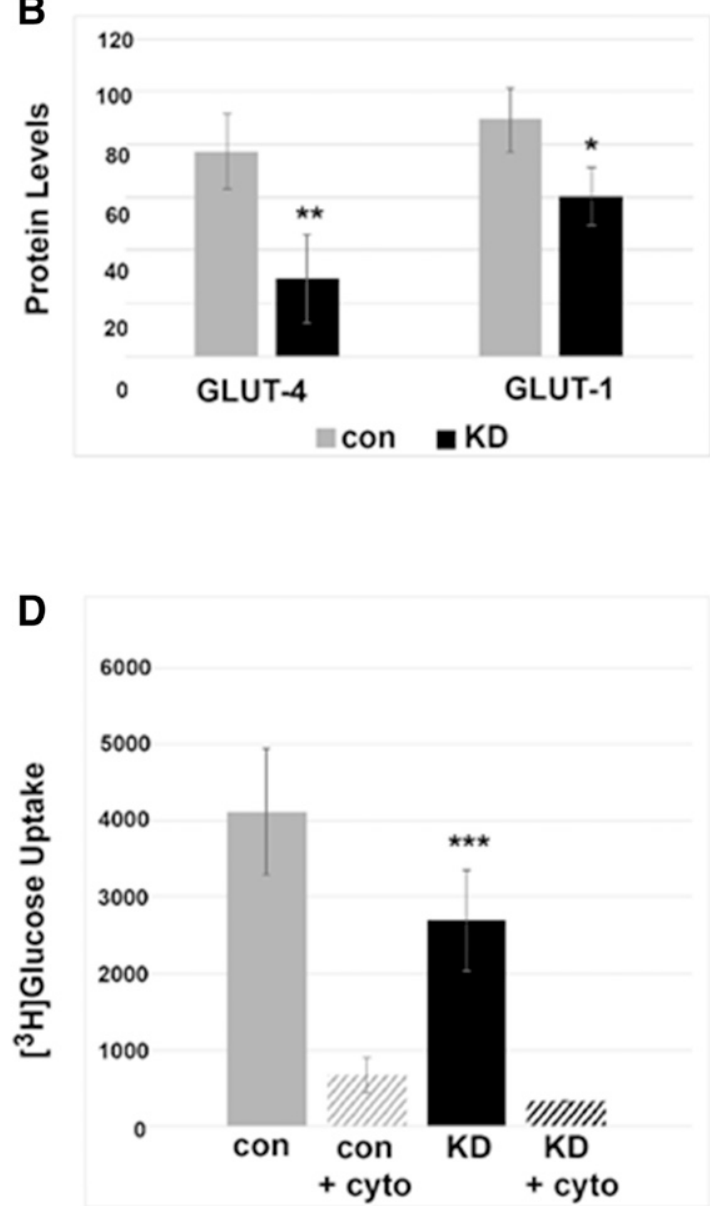

Fig. 6. PGRMC1 increases glucose uptake. (A) Western blot analysis of plasma membrane (PM) protein levels from control (con) and PGRMC1knockdown cells (KD, lanes 2 and 4) labeled with biotin and purified by avidin agarose. (B) Graphical representation of reduced GLUT-4 and GLUT-1 levels at the plasma membrane in three independent replicates of PGRMC1-knockdown cells $(P<0.01 ; P<0.05)$. (C) Western blot analysis of total GLUT-1 and GLUT-4 levels did not change in control versus knockdown cells. (D) Radioactive glucose uptake assay in control and PGRMC1-knockdown cells. Knockdown of PGRMC1 significantly reduced the uptake of ${ }^{3} \mathrm{H}$-glucose in A549 cells $(P<0.001)$ in three independent replicates. Cytochalasin B served as a negative control (inhibits glucose transport). The experiments in (B and D) are independent of each other. ${ }^{*} P<0.05 ; * * P<0.01 ; * * * P<0.001$ compared with the vehicle-treated group.

The pathways controlling IR plasma membrane trafficking are less well characterized than some other receptors, such as EGFR. However, key proteins include limb region domain containing 1 (Tseng et al., 2013), protein kinase $\mathrm{C} \varepsilon$ (Pedersen et al., 2013), and the adaptor protein growth factor receptorbound protein 10 (Morrione, 2000; Morcavallo et al., 2014). IR is ubiquitinated and associates with the ubiquitin ligase NEDD4 via growth factor receptor-bound protein 10 and with the E3 ubiquitin ligase mitsugumin 53 (Song et al., 2013) and adaptor protein with pleckstrin homology and Src homology 2 domain B adaptor protein 2 (Kishi et al., 2007). This group of proteins affects the internalization and degradation of IR, likely with variations between the two IR isoforms, in different tissues and with different types of ligand stimulation (Morcavallo et al., 2014). An important future direction of the research will be to identify PGRMC1-interacting partners in regulating IR trafficking.

Given that PGRMC1 elevated the plasma membrane levels of IR, it is counterintuitive that PGRMC1 decreased IR signaling. However, both AG205 treatment of HUH7 liver cancer cells and genetic attenuation of PGRMC1 in A549 cells elevated AKT-Ser ${ }^{473}$ phosphorylation after insulin treatment.
The results support a model in which PGRMC1 increases IR levels at the plasma membrane, but suppresses downstream signaling. Treatment with AG205 increased AKT phosphorylation, even in the absence of insulin, and this increase was completely blocked by cotreatment with the inhibitor linsitinib. Like many kinase inhibitors, linsitinib has targets other than IR, and we cannot exclude the possibility that linsitinib targets kinases other than IR that are AG205-sensitive. Notably, erlotinib did not inhibit AG205-induced AKT phosphorylation, suggesting that EGFR is not a key target in this setting. One possible explanation for the PGRMC1-IR interaction in signaling is that PGRMC1 delays IR at the plasma membrane after ligand binding, suppressing its activity as an intracellular complex. PGRMC1 homologs have been previously implicated in endocytosis (Hand et al., 2003), and a future direction of the research is to explore the mechanisms through which PGRMC1 might alter receptor endocytosis.

PGRMC1 elevated the plasma membrane levels of two glucose transporters and increased glucose transport. Mammalian glucose transport is mediated by a family of membrane glycoproteins, including GLUT-1 and GLUT-4 (Kraft et al., 2015). The GLUT-1 isoform is ubiquitously expressed and 
facilitates basal glucose uptake and transport across blood tissue barriers, whereas the GLUT- 4 isoform is predominately found in the muscle, fat, and heart tissues and mediates the rate-limiting step in regulated transport in these tissues (Zhao and Keating, 2007). The concentration of GLUT-4 at the cell surface and duration for which the protein is maintained at the surface govern the rate of glucose transport into fat and muscle cells (Chang et al., 2004). It is unclear whether increased GLUT-4 plasma membrane levels in our experiments occur through activated IR, and attempts to measure glucose uptake with insulin stimulation were technically not feasible.

We have found that PGRMC1 directly associates with IR, maintains IR at the plasma membrane, and increases insulin binding and glucose uptake in cancer cells. Both genetic manipulation and the PGRMC1 inhibitor AG205 altered IR at the plasma membrane in cell lines from different tissue types. We have previously demonstrated PGRMC1 levels in the plasma samples from cancer patients, and the present findings suggest the possibility that PGRMC1 may associate with IR in the bloodstream. If it were true, the plasma PGRMC1-IR complex could alter the levels of free insulin in the bloodstream, impacting glucose metabolism. A future direction of the research will be to determine the systemic activity of AG205 and other potential PGRMC1 ligands. However, the present studies suggest that PGRMC1 may be an important metabolic regulator with the potential to target signaling in cancer.

\section{Acknowledgments}

The authors thank Ling Jin for expert technical assistance, Drs. Brett Spear and Olivier Thibault for advice and reagents, Thomas Wilkop and Chris Richards of the University of Kentucky Imaging Core Facility for help with imaging, and Camille Blake Kaserman and Colin Rogers of Nikon Instruments for expertise in microscopy analysis.

\section{Authorship Contributions}

Participated in research design: Hampton, Anderson, Thibault, Craven.

Conducted experiments: Hampton, Anderson, Frazier.

Contributed new reagents or analytic tools: Hampton, Craven.

Performed data analysis: Hampton, Anderson.

Wrote or contributed to the writing of the manuscript: Hampton, Craven.

\section{References}

Ahmed IS, Rohe HJ, Twist KE, and Craven RJ (2010a) Pgrmc1 (progesterone receptor membrane component 1) associates with epidermal growth factor receptor and regulates erlotinib sensitivity. $J$ Biol Chem 285:24775-24782.

Ahmed IS, Rohe HJ, Twist KE, Mattingly MN, and Craven RJ (2010b) Progesterone receptor membrane component 1 (Pgrmc1): a heme-1 domain protein that promotes tumorigenesis and is inhibited by a small molecule. J Pharmacol Exp Ther $\mathbf{3 3 3}$ $564-573$.

Aizen J and Thomas P (2015) Role of Pgrmc1 in estrogen maintenance of meiotic arrest in zebrafish oocytes through Gper/Egfr. J Endocrinol 225:59-68.

Alghamdi F, Guo M, Abdulkhalek S, Crawford N, Amith SR, and Szewczuk MR (2014) A novel insulin receptor-signaling platform and its link to insulin resistance and type 2 diabetes. Cell Signal 26:1355-1368.

Bashour NM and Wray S (2012) Progesterone directly and rapidly inhibits GnRH neuronal activity via progesterone receptor membrane component 1 . Endocrinology 153:4457-4469.

Belfiore A, Frasca F, Pandini G, Sciacca L, and Vigneri R (2009) Insulin receptor isoforms and insulin receptor/insulin-like growth factor receptor hybrids in physiology and disease. Endocr Rev 30:586-623.

Boothe T, Lim GE, Cen H, Skovs $\emptyset$ S, Piske M, Li SN, Nabi IR, Gilon P, and Johnson JD (2016) Inter-domain tagging implicates caveolin-1 in insulin receptor trafficking and Erk signaling bias in pancreatic beta-cells. Mol Metab 5:366-378.

Cahill MA (2007) Progesterone receptor membrane component 1: an integrative review. J Steroid Biochem Mol Biol 105:16-36.
Chang L, Chiang SH, and Saltiel AR (2004) Insulin signaling and the regulation of glucose transport. Mol Med 10:65-71.

Crudden G, Loesel R, and Craven RJ (2005) Overexpression of the cytochrome p450 activator hpr6 (heme-1 domain protein/human progesterone receptor) in tumors. Tumour Biol 26:142-146.

de la Monte SM (2012) Brain insulin resistance and deficiency as therapeutic targets in Alzheimer's disease. Curr Alzheimer Res 9:35-66.

Elbein SC, Kern PA, Rasouli N, Yao-Borengasser A, Sharma NK, and Das SK (2011) Global gene expression profiles of subcutaneous adipose and muscle from glucosetolerant, insulin-sensitive, and insulin-resistant individuals matched for BMI. Diabetes 60:1019-1029.

Forest A, Amatulli M, Ludwig DL, Damoci CB, Wang Y, Burns CA, Donoho GP, Zanella N, Fiebig HH, Prewett MC, et al. (2015) Intrinsic resistance to cixutumumab is conferred by distinct isoforms of the insulin receptor. Mol Cancer Res 13:1615-1626.

Franks SE, Jones RA, Briah R, Murray P, and Moorehead RA (2016) BMS-754807 is cytotoxic to non-small cell lung cancer cells and enhances the effects of platinum chemotherapeutics in the human lung cancer cell line A549. BMC Res Notes 9:134.

Frasca F, Pandini G, Scalia P, Sciacca L, Mineo R, Costantino A, Goldfine ID, Belfiore A, and Vigneri R (1999) Insulin receptor isoform A, a newly recognized, highaffinity insulin-like growth factor II receptor in fetal and cancer cells. Mol Cell Biol 19:3278-3288.

Giorgino F, Belfiore A, Milazzo G, Costantino A, Maddux B, Whittaker J, Goldfine ID, and Vigneri R (1991) Overexpression of insulin receptors in fibroblast and ovary cells induces a ligand-mediated transformed phenotype. Mol Endocrinol 5: $452-459$.

Goh LK and Sorkin A (2013) Endocytosis of receptor tyrosine kinases. Cold Spring Harb Perspect Biol 5:a017459.

Guo M, Zhang C, Wang Y, Feng L, Wang Z, Niu W, Du X, Tang W, Li Y, Wang C, et al. (2016) Progesterone receptor membrane component 1 mediates progesteroneinduced suppression of oocyte meiotic prophase I and primordial folliculogenesis. Sci Rep 6:36869.

Hampton KK and Craven RJ (2014) Pathways driving the endocytosis of mutant and wild-type EGFR in cancer. Oncoscience 1:504-512.

Hand RA, Jia N, Bard M, and Craven RJ (2003) Saccharomyces cerevisiae Dap1p, a novel DNA damage response protein related to the mammalian membraneassociated progesterone receptor. Eukaryot Cell 2:306-317.

Huang S and Czech MP (2007) The GLUT4 glucose transporter. Cell Metab 5: $237-252$

Hughes AL, Powell DW, Bard M, Eckstein J, Barbuch R, Link AJ, and Espenshade PJ (2007) Dap1/PGRMC1 binds and regulates cytochrome P450 enzymes. Cell Metab 5:143-149.

Jones DR, Keune WJ, Anderson KE, Stephens LR, Hawkins PT, and Divecha N (2014) The hexosamine biosynthesis pathway and O-GlcNAcylation maintain insulin-stimulated PI3K-PKB phosphorylation and tumour cell growth after shortterm glucose deprivation. FEBS $J$ 281:3591-3608.

Kabe Y, Nakane T, Koike I, Yamamoto T, Sugiura Y, Harada E, Sugase K, Shimamura T, Ohmura M, Muraoka K, et al. (2016) Haem-dependent dimerization of PGRMC1/sigma-2 receptor facilitates cancer proliferation and chemoresistance. Nat Commun 7:11030.

Kishi K, Mawatari K, Sakai-Wakamatsu K, Yuasa T, Wang M, Ogura-Sawa M, Nakaya Y, Hatakeyama S, and Ebina Y (2007) APS-mediated ubiquitination of the insulin receptor enhances its internalization, but does not induce its degradation. Endocr $J$ 54:77-88.

Kraft TE, Hresko RC, and Hruz PW (2015) Expression, purification, and functional characterization of the insulin-responsive facilitative glucose transporter GLUT4. Protein Sci 24:2008-2019.

Krebs CJ, Jarvis ED, Chan J, Lydon JP, Ogawa S, and Pfaff DW (2000) A membraneassociated progesterone-binding protein, $25-\mathrm{Dx}$, is regulated by progesterone in brain regions involved in female reproductive behaviors. Proc Natl Acad Sci USA 97:12816-12821.

Lodde V and Peluso JJ (2011) A novel role for progesterone and progesterone receptor membrane component 1 in regulating spindle microtubule stability during rat and human ovarian cell mitosis. Biol Reprod 84:715-722.

Malaguarnera R and Belfiore A (2011) The insulin receptor: a new target for cancer therapy. Front Endocrinol (Lausanne) 2:93.

McClain DA (1992) Mechanism and role of insulin receptor endocytosis. Am J Med Sci 304:192-201.

Mir SU, Jin L, and Craven RJ (2012) Neutrophil gelatinase-associated lipocalin (NGAL) expression is dependent on the tumor-associated sigma-2 receptor S2RPgrmc1. J Biol Chem 287:14494-14501.

Moller DE, Yokota A, Caro JF, and Flier JS (1989) Tissue-specific expression of two alternatively spliced insulin receptor mRNAs in man. Mol Endocrinol 3: 1263-1269.

Morcavallo A, Stefanello M, Iozzo RV, Belfiore A, and Morrione A (2014) Ligandmediated endocytosis and trafficking of the insulin-like growth factor receptor I and insulin receptor modulate receptor function. Front Endocrinol (Lausanne) 5: 220 .

Morrione A (2000) Grb10 proteins in insulin-like growth factor and insulin receptor signaling (review). Int $J$ Mol Med 5:151-154.

Mosthaf L, Grako K, Dull TJ, Coussens L, Ullrich A, and McClain DA (1990) Functionally distinct insulin receptors generated by tissue-specific alternative splicing. EMBO J 9:2409-2413.

Nölte I, Jeckel D, Wieland FT, and Sohn K (2000) Localization and topology of ratp28, a member of a novel family of putative steroid-binding proteins. Biochim Biophys Acta 1543:123-130

Oda S, Nakajima M, Toyoda Y, Fukami T, and Yokoi T (2011) Progesterone receptor membrane component 1 modulates human cytochrome P450 activities in an isoform-dependent manner. Drug Metab Dispos 39:2057-2065. 
Papa V, Pezzino V, Costantino A, Belfiore A, Giuffrida D, Frittitta L, Vannelli GB, Brand R, Goldfine ID, and Vigneri R (1990) Elevated insulin receptor content in human breast cancer. $J$ Clin Invest 86:1503-1510.

Pedersen DJ, Diakanastasis B, Stöckli J, and Schmitz-Peiffer C (2013) Protein kinase $\mathrm{C} \varepsilon$ modulates insulin receptor localization and trafficking in mouse embryonic fibroblasts. PLoS One 8:e58046.

Peluso JJ (2013) Progesterone receptor membrane component 1 and its role in ovarian follicle growth. Front Neurosci 7:99.

Peluso JJ, Pappalardo A, Losel R, and Wehling M (2005) Expression and function of PAIRBP1 within gonadotropin-primed immature rat ovaries: PAIRBP1 regulation of granulosa and luteal cell viability. Biol Reprod 73:261-270.

Peluso JJ, Yuan A, Liu X, and Lodde V (2013) Plasminogen activator inhibitor 1 RNA-binding protein interacts with progesterone receptor membrane component 1 to regulate progesterone's ability to maintain the viability of spontaneously immortalized granulosa cells and rat granulosa cells. Biol Reprod 88:20.

Pessin JE, Thurmond DC, Elmendorf JS, Coker KJ, and Okada S (1999) Molecular basis of insulin-stimulated GLUT4 vesicle trafficking. Location! Location! Location! J Biol Chem 274:2593-2596.

Piel RB, III, Shiferaw MT, Vashisht AA, Marcero JR, Praissman JL, Phillips JD, Wohlschlegel JA, and Medlock AE (2016) A novel role for progesterone receptor membrane component 1 (PGRMC1): a partner and regulator of ferrochelatase. Biochemistry 55:5204-5217.

Posner BI (2017) Insulin signalling: the inside story. Can J Diabetes 41:108-113.

Saltiel AR and Kahn CR (2001) Insulin signalling and the regulation of glucose and lipid metabolism. Nature 414:799-806.

Sciacca L, Cassarino MF, Genua M, Vigneri P, Giovanna Pennisi M, Malandrino P, Squatrito S, Pezzino V, and Vigneri R (2014) Biological effects of insulin and its analogs on cancer cells with different insulin family receptor expression. J Cell Physiol 229:1817-1821.

Song R, Peng W, Zhang Y, Lv F, Wu HK, Guo J, Cao Y, Pi Y, Zhang X, Jin L, et al. (2013) Central role of E3 ubiquitin ligase MG53 in insulin resistance and metabolic disorders. Nature 494:375-379.

Stöckli J, Fazakerley DJ, and James DE (2011) GLUT4 exocytosis. J Cell Sci 124 4147-4159.
Sun F, Nguyen T, Jin X, Huang R, Chen Z, Cunningham RL, Singh M, and Su C (2016) Pgrmc1/BDNF signaling plays a critical role in mediating glia-neuron cross talk. Endocrinology 157:2067-2079.

Szczesna-Skorupa E and Kemper B (2011) Progesterone receptor membrane component 1 inhibits the activity of drug-metabolizing cytochromes $\mathrm{P} 450$ and binds to cytochrome P450 reductase. Mol Pharmacol 79:340-350.

Thomas P, Pang Y, and Dong J (2014) Enhancement of cell surface expression and receptor functions of membrane progestin receptor $\alpha(\mathrm{mPR} \alpha)$ by progesterone receptor membrane component 1 (PGRMC1): evidence for a role of PGRMC1 as an adaptor protein for steroid receptors. Endocrinology $\mathbf{1 5 5}$ 1107-1119.

Tseng LT, Lin CL, Tzen KY, Chang SC, and Chang MF (2013) LMBD1 protein serves as a specific adaptor for insulin receptor internalization. $J$ Biol Chem 288: 32424-32432.

Vincent EE, Elder DJ, Curwen J, Kilgour E, Hers I, and Tavaré JM (2013) Targeting non-small cell lung cancer cells by dual inhibition of the insulin receptor and the insulin-like growth factor-1 receptor. PLoS One 8:e66963.

Xu J, Zeng C, Chu W, Pan F, Rothfuss JM, Zhang F, Tu Z, Zhou D, Zeng D, Vangveravong $\mathrm{S}$, et al. (2011) Identification of the PGRMC1 protein complex as the putative sigma-2 receptor binding site. Nat Commun 2:380.

Zhang M, Robitaille M, Showalter AD, Huang X, Liu Y, Bhattacharjee A, Willard FS, Han J, Froese S, Wei L, et al. (2014) Progesterone receptor membrane component 1 is a functional part of the glucagon-like peptide-1 (GLP-1) receptor complex in pancreatic $\beta$ cells. Mol Cell Proteomics 13:3049-3062.

Zhao FQ and Keating AF (2007) Functional properties and genomics of glucose transporters. Curr Genomics 8:113-128.

Address correspondence to: Dr. Rolf J. Craven, Department of Pharmacology and Nutritional Sciences, Graduate Center for Nutritional Sciences, University of Kentucky College of Medicine, Lexington, KY 40515. E-mail: rolf. craven@uky.edu 